\title{
Diet quality and mental health in subsequent years among Canadian youth
}

\author{
Seanna E McMartin ${ }^{1}$, Stefan Kuhle ${ }^{1}$, lan Colman ${ }^{1}$, Sara FL Kirk ${ }^{2}$ and \\ Paul J Veugelers ${ }^{1, *}$ \\ ${ }^{1}$ School of Public Health, University of Alberta, 650 University Terrace, 8303-112 Street, Edmonton, Alberta, \\ Canada, T6G 2T4: ${ }^{2}$ School of Health Administration, Dalhousie University, Halifax, Nova Scotia, Canada
}

Submitted 17 May 2011: Accepted 20 January 2012: First published online 14 March 2012

\begin{abstract}
Objective: To examine the association between diet quality and the diagnosis of an internalizing disorder in children and adolescents.

Design: A prospective study examining the relationship between diet quality and mental health. FFQ responses of 3757 children were used to calculate a composite score for diet quality and its four components: variety, adequacy, moderation and balance. Physicians' diagnoses on internalizing disorders were obtained by linking the children's dietary information to administrative health data. Negative binomial regression models were used to examine the association between diet quality and diagnosis of an internalizing disorder.

Setting: The Canadian province of Nova Scotia.

Subjects: A provincially representative sample of grade 5 students (age 10-11 years). Results: Diet quality was not found to be associated with internalizing disorder in a statistically significant manner (incidence rate ratio $=1 \cdot 09 ; 95 \% \mathrm{CI} 0 \cdot 73,1 \cdot 63$ ). However, relative to children with little variety in their diets, children with greater variety in their diet had statistically significant lower rates of internalizing disorder in subsequent years (incidence rate ratio $=0 \cdot 45 ; 95 \%$ CI $0 \cdot 25,0 \cdot 82$ ).

Conclusions: These findings suggest the importance of variety in children's diet and opportunities in the prevention of adolescent depression and anxiety.
\end{abstract}

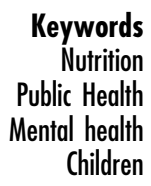

Recent research suggests a link between nutrition and mental health, particularly in relation to specific nutrients such as fatty acids ${ }^{(1,2)}$ and B vitamins ${ }^{(3)}$. However, individuals do not consume isolated nutrients. Research focusing on intakes of specific nutrients ignores the synergistic effects between nutrients ${ }^{(4)}$. There is emerging research examining the overall diet and mental health ${ }^{(5,6)}$. Studies from the $\mathrm{UK}^{(5)}$ and Spain ${ }^{(6)}$ have linked depression to poor dietary patterns in older populations. A study comparing the effect of consuming a diet with a high intake of whole foods $v$. processed foods found that individuals consuming a diet high in processed foods had greater odds of depression at the end of the study ${ }^{(5)}$. In 2004, the Canadian Community Health Survey found that the majority of Canadian youth were not receiving adequate nutrition ${ }^{(7)}$. Of all children aged 4 to 8 years, $70 \%$ were not consuming the recommended number of servings of vegetables and fruits and one-third did not meet the recommendations for milk products ${ }^{(7)}$. The growing number of children with inadequate nutrition raises public health concerns for their mental health later in life if an association exists. In the present study we examined the relationship of diet quality of children with their mental health in subsequent years.

\section{Methods}

\section{Study design}

The present study used survey data on diet and socioeconomic background from 5th grade students participating in the Children's Lifestyle and School Performance study (CLASS) in the Canadian province of Nova Scotia. This information was linked with administrative health data to obtain information on physician diagnosis of internalizing disorders in subsequent years.

\section{Study population}

CLASS was a cross-sectional, province-wide survey among grade 5 students (age 10-11 years) and their parents conducted in 2003. Approximately $97 \%$ of students in Nova Scotia attend public schools. Of all 291 public schools with grade 5 students, 282 participated by providing their grade 5 students study documentation to take home. This documentation included a consent form and survey on socio-economic background for parents to complete. Parents were also asked to provide their child's Health Insurance Number to allow for linkage of the survey data with administrative health-care databases. 
Teams of two well-trained research assistants visited each school to administer a questionnaire on activities and a modified version of the Harvard Youth/Adolescent Food Frequency Questionnaire (YAQ) ${ }^{(8)}$. The YAQ was modified slightly to include Canadian food and product names. We also omitted questions on alcohol use as our survey population was primarily 10 and 11 years of age ${ }^{(9)}$. More details on the survey are available elsewhere ${ }^{(10)}$.

\section{Administrative bealth data}

In Canada, a publicly funded health-care system provides Canadians with universal access to all medically necessary hospital and physician services ${ }^{(11)}$. The administrative health data sets encompass the Medical Services Insurance (MSI) database and the Canadian Institute for Health Information Discharge Abstract Database (CIHI DAD). Data were available from 1992 (when the child was born) until 2006. The MSI database is administered by Medavie Blue Cross for the province of Nova Scotia and contains administrative records for each insured health service rendered by a physician (including emergency room visits) and paid for by the Nova Scotia provincial health-care system. The CIHI DAD contains a comprehensive administrative transcription of each admission to a Nova Scotia hospital facility. Both of these databases contain individual patient-level information including patient demography (age, gender, location, etc.), attending physicians, diagnoses and procedures performed, service transfers while in hospital, specialty services received (excluding physiotherapy, occupational therapy) and case complexity (excluding resource intensity weight). A combination of deterministic and probabilistic matching was used to link the CLASS data with the administrative health data sets. The administrative health data sets were linked with the CLASS data through the health card numbers provided by the students' parents. Where no direct match was found, the birth date and civic address were used to establish a probable match.

\section{Exposure of interest: overall diet quality}

Nutrient intake and dietary habits were examined using the $\mathrm{YAQ}^{(8)}$. This questionnaire is used to assess usual dietary intake over the previous 12 months. It is a selfadministered tool designed for children and adolescents aged 9 to 18 years, and has been shown to be a valid measure of nutritional information among this age group ${ }^{(8)}$.

On the basis of students' responses to the YAQ and the Canadian Nutrient Files ${ }^{(12)}$ we calculated nutrient intake, numbers of daily servings of vegetables and fruits, number of daily servings of fish and daily energy intake. On the basis of these quantities we calculated the Diet Quality Index-International (DQI-I), an index that has been proved effective at identifying diet quality by incorporating many different dietary components ${ }^{(13,14)}$. The DQI-I ranges from 0 to 100, with higher scores indicating a higher diet quality, and constitutes four component scores: (i) variety; (ii) adequacy; (iii) moderation; and (iv) balance ${ }^{(13)}$. DQI-I variety assesses whether foods consumed come from diverse sources within and between food groups ${ }^{(13)}$. DQI-I adequacy allows for the evaluation of undernutrition by assessing the intake of foods that are required to ensure a healthy diet ${ }^{(13)}$. Conversely, DQI-I moderation assesses overnutrition by evaluating intakes of foods that need restriction due to their association with chronic diseases $^{(13)}$. Lastly, DQI-I balance examines the proportion of intakes from different energy sources ${ }^{(13)}$. In addition to assessing the importance of diet quality and its four composites for mental health, we explored the association of food items and nutrients that other studies had implicated or suggested in the causation of mental health disorders. These included vegetable and fruit consumption, fish consumption ${ }^{(1,15)}$, vitamin $\mathrm{B}_{6}{ }^{(3)}$, folate ${ }^{(3)}$, vitamin $\mathrm{B}_{12}{ }^{(16)}$ and $n-3$ fatty acids ${ }^{(1,2)}$. With respect to the later, we evaluated both the absolute amount of $n$ - 3 fatty acids as well as the ratio of $n-3: n-6$ fatty acids ${ }^{(1,2)}$.

\section{Outcome of interest: internalizing disorder}

The primary outcome of interest was the number of health-care provider contacts where a primary diagnosis of an internalizing disorder was given between 2003 and 2006 for each child. Internalizing disorders include common symptoms of depression and anxiety such as low mood, inhibition, excessive worrying, physical complaints, trouble sleeping and shyness ${ }^{(17)}$. A child was considered to have an internalizing disorder if he/she received a diagnosis, according to the International Classification of Diseases, ninth revision (ICD-9) or tenth revision (ICD-10), of a depressive episode, recurrent or persistent mood disorder, neurotic or general anxiety disorder, an acute reaction to severe stress, or an emotional disorder with onset specific to childhood (see Table 1 for list of ICD-9/10 codes used).

\section{Covariates}

Analyses were adjusted for factors that could potentially confound the relationship between diet quality and

Table 1 ICD-9/10 codes identifying diagnosis of internalizing disorder

\begin{tabular}{lcc}
\hline Disorder & ICD-9 code & ICD-10 code \\
\hline $\begin{array}{l}\text { Depressive episode, recurrent/persistent or unspecified mood } \\
\text { disorder (excluding bipolar) }\end{array}$ & $296 \cdot 2,296 \cdot 3,296 \cdot 9,311$ & F32, F33, F34, F38, F39 \\
$\begin{array}{l}\text { Neurotic, general anxiety disorders, reaction to severe stress, } \\
\text { emotional disorders with onset specific to childhood }\end{array}$ & $300,308,309,313$ & F40, F41, F42, F43, F48, \\
\hline
\end{tabular}

ICD-9, International Classification of Diseases, ninth revision; ICD-10, International Classification of Diseases, tenth revision.

Source: http://en.wikipedia.org/wiki/List_of_ICD-9_codes and http://en.wikipedia.org/wiki//CD-10 (accessed February 2012). 
internalizing disorders. These included: energy intake, gender, household income, parental marital status and education, body weight status, physical activity level and area of residence. The parental survey provided information on sociodemographic characteristics including household income $(23.3 \%$ missing), parental education ( $7 \cdot 1 \%$ missing), parental marital status and urban/rural residence. Urban and rural residence was classified based on the postal code of the student's home address obtained from the parents ${ }^{(18)}$. Body weight status was defined using the age- and gender-specific BMI cut-off points for children and adolescents established by the International Obesity Task Force and based on healthrelated adult definitions of overweight $\left(\geq 25 \mathrm{~kg} / \mathrm{m}^{2}\right)$ and obesity $\left(\geq 30 \mathrm{~kg} / \mathrm{m}^{2}\right)$. Student height was measured to the nearest $0 \cdot 1 \mathrm{~cm}$ after students had removed their shoes, and body weight was measured to the nearest $0 \cdot 1 \mathrm{~kg}$ on calibrated digital scales. Physical activity level was determined from the student survey, which included validated questions taken from the National Longitudinal Survey for Children and Youth ${ }^{(19)}$. These questions asked students to indicate how often they participated in organized sports and leisure-time physical activities per week ${ }^{(19)}$. Total energy intake was adjusted for as per recommendations for the analyses of food frequency data ${ }^{(20)}$.

\section{Number of participants}

Of the 5517 grade 5 students who received parental consent to participate, 5200 students completed the surveys. The average participation rate per school was $51 \cdot 1 \%$. Of the 5200 students who participated, 4380 had adequate information to be linked with the administrative healthcare databases. Among these, 3953 students had complete information from the FFQ and an energy intake of more than $2092 \mathrm{~kJ} / \mathrm{d}(500 \mathrm{kcal} / \mathrm{d})$ and less than $20920 \mathrm{~kJ} / \mathrm{d}$ $(5000 \mathrm{kcal} / \mathrm{d})$. Energy intakes of less than $2092 \mathrm{~kJ} / \mathrm{d}$ and more than $20920 \mathrm{~kJ} / \mathrm{d}$ are considered outliers and should be excluded as per recommendations for the analysis of food frequency data ${ }^{(21)}$. Children with a diagnosis of an internalizing disorder before the administration of the survey in 2003 ( $n$ 196) were excluded from the analysis, leaving a final sample of 3757 students. Among these students $88 \%$ had at least one health-care provider contact in 2005 or 2006.

\section{Statistical analysis}

The association between diet quality and the diagnosis of internalizing disorder was examined using multilevel regression models to accommodate the nested structure of the data whereby observations of students are nested within schools. The distribution of the number of healthcare provider contacts with a diagnosis of an internalizing disorder showed overdispersion (i.e. the variance of the data exceeded what might be expected under the Poisson distribution). Therefore, negative binomial regression was used instead of Poisson regression. Regression results are reported as incidence rate ratios (IRR). IRR for diet quality were adjusted for energy intake, gender, household income, parental marital status and education, body weight status, physical activity level and geographic area. Missing values for these variables were considered as separate covariate categories in the regression analyses, but their estimates are not presented. As CLASS participation rates in residential areas with lower estimates of household income were slightly lower than the average, response weights were calculated to overcome potential non-response bias and to yield provincial population estimates for grade 5 students in Nova Scotia ${ }^{(10)}$. The STATA/SE 11 statistical software package (Stata Corp., College Station, TX, USA) was used for the statistical analysis.

\section{Results}

The incidence of a health-care provider contact for internalizing disorder between 2003 and 2006 in our sample was $7 \cdot 8(95 \%$ CI $7 \cdot 0,8 \cdot 7) \%$ over the four years following the survey. This was slightly higher among girls ( $n$ 172) relative to boys ( $n$ 122; Table 2). A greater proportion of students diagnosed with internalizing disorder were in lower socioeconomic groups and from single-parent families. The proportion of students diagnosed with an internalizing disorder was lower among those children who were physically active and had a normal body weight (Table 2).

Table 3 presents IRR (adjusted for energy intake and fully adjusted) for the association of diet quality and its components with the number of health-care provider contacts with a diagnostic code for internalizing disorder. Diet quality was not significantly associated with internalizing disorder. Relative to children with little variety in their diets, those with greater variety in their diets had a statistically significant lower rate of receiving a diagnosis of internalizing disorder. Relative to children in the lowest tertile for diet variety, children in the highest tertile for variety had approximately half as many visits with a diagnosis of internalizing disorder. Relative to children in the lowest level for diet adequacy, those in the highest tertile for adequacy demonstrated somewhat lower rates of being diagnosed with internalizing disorder, but these differences were not statistically significant. Dietary moderation and balance showed no statistically significant associations with internalizing disorders.

As shown in Table 4, none of the food items and nutrients including vegetable and fruit consumption, vitamin $\mathrm{B}_{6}$, folate, vitamin $\mathrm{B}_{12}, n-3$ fatty acids and $n-3: n-6$ fatty acid ratio showed a statistically significant association with internalizing disorder. However, fish consumption did reveal a statistically significant finding. Relative to the one-third of children with the lowest fish consumption, the one-third of children with the highest fish consumption had lower rates of being diagnosed with internalizing disorder (IRR $=0.59 ; 95 \%$ CI $0 \cdot 41,0 \cdot 87)$. 
Table 2 Sociodemographic and health characteristics of participants: grade 5 students (age 10-11 years), Children's Lifestyle and School Performance study (CLASS), Nova Scotia, Canada, 2003

\begin{tabular}{|c|c|c|}
\hline Characteristic & $\begin{array}{l}\text { Proportion of } \\
\text { total population } \\
(\%)\end{array}$ & $\begin{array}{c}\text { Incidence of } \\
\text { internalizing } \\
\text { disorder* (\%) }\end{array}$ \\
\hline \multicolumn{3}{|l|}{ Gender } \\
\hline Female & 52 & $8 \cdot 7$ \\
\hline Male & 48 & $6 \cdot 8$ \\
\hline \multicolumn{3}{|l|}{ Household income (\$CAN) } \\
\hline$<20000$ & 11 & $11 \cdot 0$ \\
\hline $20001-40000$ & 23 & $9 \cdot 6$ \\
\hline $40001-60000$ & 27 & $7 \cdot 3$ \\
\hline$>60000$ & 39 & $6 \cdot 2$ \\
\hline \multicolumn{3}{|l|}{ Parental education } \\
\hline Secondary school or less & 31 & $7 \cdot 5$ \\
\hline College & 38 & $7 \cdot 9$ \\
\hline University & 31 & $8 \cdot 0$ \\
\hline \multicolumn{3}{|l|}{ Parental marital status } \\
\hline Married or common law & 83 & $7 \cdot 2$ \\
\hline $\begin{array}{l}\text { Separated/divorced/ } \\
\text { widowed/single }\end{array}$ & 17 & $11 \cdot 0$ \\
\hline \multicolumn{3}{|l|}{ Residence } \\
\hline Urban & 66 & $7 \cdot 2$ \\
\hline Rural & 34 & $11 \cdot 0$ \\
\hline \multicolumn{3}{|l|}{ Physical activity level } \\
\hline 2 times/week or less & 23 & $10 \cdot 7$ \\
\hline$>2$ to 4 times/week & 17 & $8 \cdot 1$ \\
\hline$>4$ to 7 times/week & 34 & $7 \cdot 1$ \\
\hline$>7$ times/week & 26 & $6 \cdot 2$ \\
\hline \multicolumn{3}{|l|}{ Body weight status } \\
\hline Normal weight & 67 & $7 \cdot 4$ \\
\hline Overweight, not obese & 23 & $7 \cdot 7$ \\
\hline Obese & 10 & $10 \cdot 8$ \\
\hline
\end{tabular}

All estimates were weighted for non-response and therefore pertain to all grade 5 students in Nova Scotia, Canada. The analyses were based on 3757 students, among whom 294 were diagnosed with an internalizing disorder in the years following the nutritional assessment.

${ }^{*} A$ participant was considered as having internalizing disorder if she or he had one or more health-care provider contacts with a diagnosis of internalizing disorder.

\section{Discussion}

The present study is one of the few investigating the relationship between diet quality and internalizing disorders in children and adolescents. Our findings suggest that overall diet quality does not appear to play a major role in the risk of developing an internalizing disorder and seem to contradict findings from previous stu$\operatorname{dies}^{(5,6,22)}$. Two of these studies had also applied an FFQ but had longer durations of follow-up ${ }^{(5,6)}$.

Although overall diet quality did not show a significant relationship, dietary variety was associated with internalizing disorder in subsequent years. Dietary variety is described as the consumption of foods from diverse sources within and between food groups ${ }^{(13)}$. This suggests that it is important to expose children to a variety of foods in order to meet the recommended number of servings from each food group. Having diversity in the diet has been shown to provide more of the important nutrients for a balanced diet among adults ${ }^{(23)}$. By exposing children to many different foods they are more likely to consume sufficient amounts of the essential nutrients to
Table 3 Incidence rate ratios with 95\% confidence intervals for the association between indicators of diet quality and number of health-care provider contacts for internalizing disorder: grade 5 students (age 10-11 years), Children's Lifestyle and School Performance study (CLASS), Nova Scotia, Canada, 2003

\begin{tabular}{lcccc}
\hline Dietary indicator & $\mathrm{IRR}^{*}$ & $95 \% \mathrm{Cl}$ & $\mathrm{IRR} \dagger$ & $95 \% \mathrm{Cl}$ \\
\hline DQI-I overall & & & & \\
$\quad$ First tertile (lowest) & 1.00 & - & 1.00 & - \\
$\quad$ Second tertile & 0.94 & $0.60,1.48$ & 0.99 & $0.69,1.44$ \\
$\quad$ Third tertile (highest) & 0.97 & $0.65,1.46$ & 1.09 & $0.73,1.63$ \\
DQI-I variety & & & & \\
$\quad$ First tertile (lowest) & 1.00 & - & 1.00 & - \\
$\quad$ Second tertile & 0.61 & $0.32,0.99$ & 0.60 & $0.40,0.89$ \\
$\quad$ Third tertile (highest) & 0.44 & $0.25,0.80$ & 0.45 & $0.25,0.82$ \\
DQI-I adequacy & & & & \\
$\quad$ First tertile (lowest) & 1.00 & - & 1.00 & - \\
$\quad$ Second tertile & 0.84 & $0.53,1.34$ & 0.89 & $0.59,1.35$ \\
$\quad$ Third tertile (highest) & 0.59 & $0.31,1.10$ & 0.64 & $0.34,1.20$ \\
DQI-I moderation & & & & \\
$\quad$ First tertile (lowest) & 1.00 & - & 1.00 & - \\
$\quad$ Second tertile & 0.88 & $0.53,1.44$ & 0.94 & $0.62,1.41$ \\
$\quad$ Third tertile (highest) & 1.02 & $0.59,1.76$ & 1.07 & $0.66,1.73$ \\
DQI-I balance & & & & \\
$\quad<1$ (poor) & 1.00 & - & 1.00 & - \\
$\quad \geq 1$ (good) & 0.93 & $0.65,1.35$ & 1.06 & $0.66,1.73$ \\
\hline
\end{tabular}

IRR, incidence rate ratio.

All estimates were weighted for non-response and therefore pertain to all grade 5 students in Nova Scotia, Canada. The analyses were based on 3757 students, among whom 294 were diagnosed with an internalizing disorder in the years following the nutritional assessment.

*Adjusted for energy intake.

tAdjusted for energy intake, gender, household income, parental marital status and education, body weight status, physical activity level and geographic area.

ensure a healthy development and, as shown in the present study, thereby lower the risk of developing internalizing disorder. Dietary moderation, which refers to the lack of overnutrition and excess intakes of foods, has been associated with the development of chronic diseases ${ }^{(13)}$. The present study, however, showed no statistically significant relationship between dietary moderation and the development of an internalizing disorder. In other words, dietary diversity rather than excess consumption seems to determine the development of mental health.

Fish is the most widely available source of $n-3$ fatty acids, an essential nutrient that has been shown to reduce the risk of depressive symptoms ${ }^{(1,2)}$. The $n-3$ fatty acids are key components of synaptic neuronal membranes and play an important role in neurotransmitter function ${ }^{(24)}$. Changes to this membrane may impair neurotransmitter function - in particular, the functioning of serotonin and dopamine $^{(24)}$ - which may explain the association between $n-3$ fatty acids and depressive symptoms. Also an imbalance between $n-3$ fatty acids and n-6 fatty acids has been linked to an increase in depression ${ }^{(25)}$. In the present study, however, we did not observe an association of $n-3$ fatty acids and the $n-3: n-6$ fatty acid ratio with internalizing disorders, but we did observe an association of fish consumption with internalizing disorders. More fish consumption would imply more $n-3$ intake, for which we did not observe an association with mental health. Fish consumption is also referred to 
Table 4 Incidence rate ratios with $95 \%$ confidence intervals for the association between food items and nutrients and number of health-care provider contacts for internalizing disorder: grade 5 students (age 10-11 years), Children's Lifestyle and School Performance study (CLASS), Nova Scotia, Canada, 2003

\begin{tabular}{|c|c|c|c|c|}
\hline Dietary indicator & $\mathrm{IRR}^{*}$ & $95 \% \mathrm{Cl}$ & IRRt & $95 \% \mathrm{Cl}$ \\
\hline \multicolumn{5}{|l|}{ Fruit and vegetable intake } \\
\hline First tertile (lowest) & $1 \cdot 00$ & - & 1.00 & - \\
\hline Second tertile & 1.06 & $0.68,1.66$ & 1.04 & $0.71,1.53$ \\
\hline Third tertile (highes & $1 \cdot 15$ & $0.73,1.81$ & $1 \cdot 25$ & $0.80,1.99$ \\
\hline \multicolumn{5}{|l|}{ Folate } \\
\hline First tertile (lowest) & $1 \cdot 00$ & - & $1 \cdot 00$ & - \\
\hline Second tertile & 1.04 & $0.64,1.69$ & $1 \cdot 11$ & $0 \cdot 73,1 \cdot 70$ \\
\hline Third tertile (highest) & $1 \cdot 18$ & $0.52,2.69$ & $1 \cdot 21$ & $0.64,2 \cdot 32$ \\
\hline \multicolumn{5}{|l|}{ Vitamin $\mathrm{B}_{6}$} \\
\hline First tertile (lowes & 1.00 & - & $1 \cdot 00$ & - \\
\hline Secc & $1 \cdot 22$ & $0.71,1.79$ & $1 \cdot 17$ & $0.77,1.77$ \\
\hline Third tertile (highest) & 0.90 & $0.48,1.72$ & $1 \cdot 05$ & $0.56,1.99$ \\
\hline \multicolumn{5}{|l|}{ Vitamin $B_{12}$} \\
\hline First tertile (lowe & $1 \cdot 00$ & - & $1 \cdot 00$ & - \\
\hline & & $\cdot 46,1$ & & $0 \cdot 52,1 \cdot 13$ \\
\hline Third tertile & & 25 & & $0.50,1.17$ \\
\hline \multicolumn{5}{|l|}{ Fish intake } \\
\hline First tertile (lowest) & $1 \cdot 00$ & - & 1.00 & - \\
\hline $\mathrm{Sec}$ & & $0.52,1$ & $0 \cdot 88$ & $0.56,1.39$ \\
\hline Third tertile (highes & 65 & 45,0 & 0.59 & $0.41,0.87$ \\
\hline \multicolumn{5}{|l|}{$n-3$ Fatty acids } \\
\hline First tertile (lowest) & $1 \cdot 00$ & - & 1.00 & - \\
\hline Second tert & $1 \cdot 17$ & $0 \cdot 59,2 \cdot 31$ & 0.81 & $0.58,1 \cdot 13$ \\
\hline Third tertile (highest) & $1 \cdot 00$ & $1 \cdot 00,1 \cdot 00$ & 0.97 & $0.61,1.55$ \\
\hline \multicolumn{5}{|l|}{$n-3: n-6$ Fatty acid ratio } \\
\hline First tertile (lowest) & $1 \cdot 00$ & - & $1 \cdot 00$ & - \\
\hline Second tertile & $0 \cdot 71$ & $0.52,0.96$ & $0 \cdot 70$ & $0.52,0.96$ \\
\hline Third tertile (highest) & 0.90 & $0.67,1 \cdot 20$ & 0.90 & $0.67,1.21$ \\
\hline \multicolumn{5}{|l|}{$\%$ Energy from fat } \\
\hline First tertile (lowest) & $1 \cdot 00$ & - & $1 \cdot 00$ & - \\
\hline Second tertile & & $0.56,1$ & 0.74 & $0.51,1.09$ \\
\hline Third tertile (highest) & 0.93 & $0.57,1.50$ & 0.82 & $0.55-1.22$ \\
\hline
\end{tabular}

IRR, incidence rate ratio.

All estimates were weighted for non-response and therefore pertain to all grade 5 students in Nova Scotia, Canada. The analyses were based on 3757 students, among whom 294 were diagnosed with an internalizing disorder in the years following the nutritional assessment.

${ }^{*}$ Adjusted for energy intake.

†Adjusted for energy intake, gender, household income, parental marital status and education, body weight status, physical activity level and geographic area.

as an indicator of dietary variety, for which we did observe an association with mental health.

The continuing increase in childhood overweight rates is acknowledged as a major public health problem as it underlies the current rises in the incidence of type 2 diabetes and other chronic diseases with mounting healthcare costs ${ }^{(26)}$. Many jurisdictions of Western countries are therefore preparing for, or are already implementing, population-based health promotion initiatives aimed to reduce the prevalence of overweight and consequent burden of chronic diseases. Previous literature has noted a relationship between body weight status and lower selfesteem among children ${ }^{(27)}$. Lower self-esteem may be an early predictor for poor mental health later in life ${ }^{(28)}$. Those population-based health promotion initiatives that also promote dietary variety may therefore benefit self-esteem and reduce internalizing disorders. These initiatives also provide unique opportunities for further study of the importance of diet for mental health and to move evidence based on cross-sectional associations to stronger evidence from intervention research.

The relatively short follow-up period of 4 years should be noted as a limitation of the present study and does not completely rule out the possibility of reverse causality. It is possible that poor mental health conditions had affected the child's dietary choices in a negative manner. Future studies would benefit from following children into early adulthood to assess the development of mental health problems over a longer duration, as the conduct of randomized controlled trials to study dietary variety is not uncomplicated. Another limitation may relate to the fact that only those children who were seen and diagnosed by a health-care professional could be considered as cases of internalizing disorder. This may represent an underestimation as children with barriers to accessing health care or avoiding treatment may have been missed.

We assessed diet quality based on responses to an FFQ that was validated and suitable for this age group. We chose to use an FFQ as our interest was in capturing 'general dietary intake' as opposed to capturing intake on a specific day or during a specific week. In addition, as we engage in public health research, we work with representative large sample sizes and thus the self-administrative nature of FFQ is essential to the feasibility of our work. A widely acknowledged limitation of FFQ is their inaccuracy with regard to estimating 'absolute intake'. In the present manuscript we avoided the analyses and presentation of 'absolute intake'. On the other hand, FFQ appropriately rank order 'dietary intake, (21). In our analytic approaches, we utilized this strength of rank ordering by making comparisons across tertiles of diet quality and tertiles of nutrient intake. A further limitation of FFQ pertains to the fact that only information on the frequency and quantity of foods from a specified list is collected and that food preparation methods are not consistently considered ${ }^{(29)}$.

Despite these potential limitations, the present study is unique in its size and had several methodological strengths: it was a population-based study with a relatively high response rate; it involved a clean cohort by excluding students previously diagnosed with internalizing disorder; and it allowed for adjustment of sociodemographic factors in the analysis. In addition, the use administrative data provided valid and reliable information and minimized bias in the assessment of internalizing disorder.

\section{Conclusions}

The present findings suggest that dietary variety in children may reduce the risk of developing internalizing disorders. This has important implications for the prevention of mental illness due to the modifiable nature of individual diet. Intervention studies and studies with longer follow-up are needed to expand on the present findings. 


\section{Acknowledgements}

Sources of funding: Financial support for administrative data linkage was provided by a Canada Foundation for Innovation Leaders Opportunity Fund award to S.F.L.K. The CLASS project was funded through a Canadian Population Health Initiative operating grant to P.J.V. The present analyses were supported through a grant by the Canadian Institutes for Health Research, The Heart and Stroke Foundation of Canada, and the Canadian Population Health Initiative to P.J.V. S.F.L.K. is supported through a Canada Research Chair in Health Services Research. P.J.V. is supported through a Canada Research Chair in Population Health and an Alberta Innovates - Health Solutions Scholarship. I.C. is a recipient of an Alberta Innovates - Health Solutions Population Health Investigator Award and a Canadian Institutes of Health Research New Investigator Award. Conflict of interest declaration: The authors have no conflict of interest. Authors' contributions: S.E.M. led in manuscript writing and contributed to the detailing of the analysis. S.K. established data linkages, conducted statistical analysis, and contributed to the conceptual design and writing of the manuscript. I.C. detailed the outcome of interest and contributed to the writing of the manuscript. S.F.L.K. contributed to the conceptual design and writing of the manuscript. P.J.V., the principal investigator of the study, contributed to the conceptual design and writing of the manuscript. Acknowledgements: The authors would like to thank Charmaine Cooke and Yan Wang from the Population Health Research Unit, Dalhousie University, for their assistance with data provision and access. The authors also thank Dr K. Storey for critiquing the study.

\section{References}

1. Murakami K, Miyake Y, Sasaki S et al. (2010) Fish and $n$-3 polyunsaturated fatty acid intake and depressive symptoms: Ryukyus Child Health Study. Pediatrics 126, 623-630.

2. Freeman MP, Hibbeln JR, Wisner KL et al. (2006) Omega-3 fatty acids: evidence basis for treatment and future research in psychiatry. J Clin Psychiatry 67, 1954-1967.

3. Murakami K, Miyake Y, Sasaki S et al. (2010) Dietary folate, riboflavin, vitamin B-6, and vitamin B-12 and depressive symptoms in early adolescence: the Ryukyus Child Health Study. Psychosom Med 72, 763-768.

4. Hu FB (2002) Dietary pattern analysis: a new direction in nutritional epidemiology. Curr Opin Lipidol 13, 3-9.

5. Akbaraly TN, Brunner EJ, Ferrie JE et al. (2009) Dietary pattern and depressive symptoms in middle age. $\mathrm{BrJ}$ Psychiatry 195, 408-413.

6. Sanchez-Villegas A, Delgado-Rodriguez M, Alonso A et al. (2009) Association of the Mediterranean dietary pattern with the incidence of depression: the Seguimiento Universidad de Navarra/University of Navarra follow-up (SUN) cohort. Arch Gen Psychiatry 66, 1090-1098.

7. Garriguet D (2006) Nutrition: Findings from the Canadian Community Health Survey. Overview of Canadians' Eating Habits. Ottawa: Statistics Canada.

8. Rockett HR, Wolf AM \& Colditz GA (1995) Development and reproducibility of a food frequency questionnaire to assess diets of older children and adolescents. J Am Diet Assoc 95, 336-340.
9. Kuhle S, Kirk S, Ohinmaa A et al. (2011) Use and cost of health services among overweight and obese Canadian children. Int J Pediatr Obes 6, 142-148.

10. Veugelers PJ \& Fitzgerald AL (2005) Effectiveness of school programs in preventing childhood obesity: a multilevel comparison. Am J Public Health 95, 432-435.

11. Health Canada (2009) Health Care System. http://www.hcsc.gc.ca/hcs-sss/index-eng.php (accessed October 2010).

12. Health Canada (2007) Food and Nutrition: The Canadian Nutrient File. http://www.hc-sc.gc.ca/fn-an/nutrition/fichenutri-data/cnf_aboutus-aproposdenous_fcen-eng.php (accessed October 2010).

13. Kim S, Haines PS, Siega-Riz AM et al. (2003) The Diet Quality Index-International (DQI-I) provides an effective tool for cross-national comparison of diet quality as illustrated by China and the United States. J Nutr 133, 3476-3484.

14. Patterson RE, Haines PS \& Popkin BM (1994) Diet quality index: capturing a multidimensional behavior. $J$ Am Diet Assoc 94, 57-64.

15. Timonen M, Horrobin D, Jokelainen J et al. (2004) Fish consumption and depression: the Northern Finland 1966 birth cohort study. J Affect Disord 82, 447-452.

16. Sanchez-Villegas A, Henriquez $\mathrm{P}$, Bes-Rastrollo $\mathrm{M}$ et al. (2006) Mediterranean diet and depression. Public Health Nutr 9, 1104-1109.

17. Goldberg D \& Goodyer I (2005) The Origins and Course of Common Mental Disorders. New York: Routledge.

18. Statistics Canada (2007) How Postal Codes Map to Geographic Areas. http://www.statcan.gc.ca/pub/92f0138m/ 92f0138m2007001-eng.pdf (accessed October 2011).

19. Statistics Canada (2010) National longitudinal survey of children and youth (NLSCY). http://www.statcan.gc.ca.login. ezproxy.library.ualberta.ca/cgi-bin/imdb/p2SV.pl?Function= getSurvey\&SDDS $=4450 \&$ lang $=$ en $\& \mathrm{db}=\mathrm{IMDB} \& \mathrm{dbg}=\mathrm{f} \& \mathrm{adm}=$ 8\&dis $=2$ (accessed February 2011).

20. Willett WC, Howe GR \& Kushi LH (1997) Adjustment for total energy intake in epidemiologic studies. Am J Clin Nutr 65, 4 Suppl., S1220-S1228.

21. Willett WC (1998) Nutritional Epidemiology, 2nd ed. New York: Oxford University Press.

22. Jacka FN, Kremer PJ, Leslie ER et al. (2010) Associations between diet quality and depressed mood in adolescents: results from the Australian Healthy Neighbourhoods Study. Aust N Z J Psychiatry 44, 435-442.

23. Foote JA, Murphy SP, Wilkens LR et al. (2004) Dietary variety increases the probability of nutrient adequacy among adults. $J$ Nutr 134, 1779-1785.

24. Salem N, Litman B, Kim HY et al. (2001) Mechanisms of action of docosahexaenoic acid in the nervous system. Lipids 36, 945-959.

25. Kiecolt-Glaser JK, Belury MA, Porter K et al. (2007) Depressive symptoms, omega-6:omega-3 fatty acids, and inflammation in older adults. Psychosom Med 69, 217-224.

26. Finucane MM, Stevens GA, Cowan MJ et al. (2011) National, regional, and global trends in body-mass index since 1980: systematic analysis of health examination surveys and epidemiological studies with 960 country-years and $9 \cdot 1$ million participants. Lancet $\mathbf{3 7 7}$, $557-567$.

27. Wang F \& Veugelers PJ (2008) Self-esteem and cognitive development in the era of the childhood obesity epidemic. Obes Rev 9, 615-623.

28. Paradise AW \& Kernis MH (2002) Self-esteem and psychological well-being: implications of fragile self-esteem. J Soc Clin Psychol 21, 345-361.

29. Thompson FE \& Byers T (1994) Dietary assessment resource manual. J Nutr 124, 11 Suppl., S2245-S2317. 